\title{
Autocuidado postural: educação em saúde com adolescentes do meio rural
}

\author{
Postural self care: health education \\ with adolescents in rural areas
}

Fisisenectus. Unochapecó

Ano 2, n. 2 - Jul/Dez. 2014 p. $35-44$

Márcia Regina da Silva. marciaf@unochapeco.edu.br

Mestre em Biociências e Reabilitação. Docente do curso de graduação em fisioterapia da da Universidade Comunitária da Região de Chapecó - Unochapecó.

Lucimare Ferraz. Iferraz@unochapeco.edu.br

Doutora em Saúde Coletiva. Docente do Programa de Pós-Graduação Strictu Sensu em Ciências da Saúde da Universidade Comunitária da Região de Chapecó - Unochapecó.

Paula Marco Marchiori. paula.marchiori@hotmail.com Mestre em Ciências da Saúde. Docente do Centro de Educação Profissionalizante (Cedup), unidade de Chapecó e do Programa Nacional de Acesso ao Ensino Técnico e Emprego (Pronatec).

Cássia Cristina Braghini.cafisio@unochapeco.edu.br

Mestre em Ciências da Saúde. Docente do Curso de Graduação em Fisioterapia da Universidade Comunitária da Região de Chapecó - Unochapecó.

Fátima Ferretti.ferrettifisio@yahoo.com.br

Doutora em Saúde Coletiva. Docente do Programa de Pós-Graduação Strictu Sensu em Ciências da Saúde da Universidade Comunitária da Região de Chapecó - Unochapecó.

Ana Paula Rech. anapaula.r@unochapeco.edu.br

Enfermeira. Especialista em Saúde da Família pela UFSC.

Jilciane Fatima Ferrazzo.jilciane@unochapeco.edu.br

Enfermeira.

\section{Resumo}

O presente estudo teve por objetivo promover o autocuidado postural aos adolescentes do meio rural, pois percebeu-se a necessidade de desenvolver ações de educação em saúde com os adolescentes do meio rural sobre os aspectos posturais para que ao se apropriarem de conhecimento possam exercer o autocuidado, bem como tornar-se um multiplicador do saber em suas famílias e comunidade. Nesse artigo buscamos rever o conceito de postura corporal; a relação problema postural, dor/desconforto musculoesquelético dos trabalhadores com a postura e movimento corporal adotado durante a jornada de trabalho e resgatamos a prática de educar em saúde considerando o aspecto cultural. Trata-se de uma pesquisa-ação, desenvolvida com 65 estudantes do sétimo e oitavo ano de duas escolas do meio rural de um município do Oeste de Santa Catarina (SC), no primeiro semestre de 2013. A análise dos dados coletados foi realizada por meio de estatística descritiva. A pesquisa demonstrou que a maioria dos estudantes apresentou hábitos posturais inadequados, destacando a postura sentada na cadeira, a forma de carregar material escolar e de levantar objetos leves e pesados do chão, fato que evidencia a importância do desenvolvimento de atividades educativas que abordem tais questões.

\section{Palavras-chave}

Adolescente; Educação em saúde; Autocuidado.

\section{Fisiłenectus}




\begin{abstract}
The present study aimed to promote self-care postural rural adolescents, because they realized the need to develop health education initiatives with rural teenagers on aspects for appropriating the posture of knowledge may exercise self-care as well as become a multiplier of knowledge in their families and community. In this article we review the concept of body posture; the relationship postural problem, musculoskeletal pain/discomfort of workers with the posture and body movement adopted during the workday and rescued the practice to educate in health considering the cultural aspect. This is an action-research, developed with 65 students in 7th and 8th year of two schools in the rural areas of a city in the West of Santa Catarina (SC), in the first half of 2013. The data was performed by means of descriptive statistics. The research showed that most students presented inadequate postural habits, highlighting the sitting posture in the Chair, the form of school supplies and loading up heavy and light objects from the ground, a fact that highlights the importance of developing educational activities that address such issues.
\end{abstract}

\title{
Keywords
}

Adolescent; Health Education; Self Care.

\section{Introdução}

$\infty \times \infty \times \infty \times \infty \times \infty \times \infty \times \infty \times \infty \times \infty \times \infty \times \infty \times \infty)$

A postura corporal é um composto das diferentes posições das articulações do corpo em um dado momento e estão relacionadas com características anatômicas, biomecânicas, hábitos posturais e interferências ambientais. A postura correta é a posição na qual um mínimo de estresse é aplicado a cada articulação ${ }^{1-2}$. A postura tem relação com o alinhamento das partes do corpo quando se está em pé, sentado ou deitado sendo necessário um equilíbrio muscular para mantê-la adequadamente, evitando sintomas de dor e desconforto ${ }^{3}$.

A infância e a adolescência são períodos em que o corpo está em fase de formação, e os cuidados posturais são fundamentais, sem os quais as alterações podem se instalar e favorecer o desenvolvimento de problemas musculoesqueléticos, principalmente na região da coluna vertebral ${ }^{4}$. A postura sentada sem o apoio lombar, o carregamento de pesos elevados e movimentos incorretos que envolvem a flexão anterior da coluna ou ainda a flexão associada à rotação e levantamento de pesos, aumentam a sobrecarga na região lombar e são fatores que podem contribuir para lesões nesse segmento ${ }^{5}$.

As queixas de desconforto, dor e problemas posturais muitas vezes são consequência de atividades repetitivas ou mantidas associadas ou não ao alinhamento postural habitualmente errado, o que é comum em atividades laborais, seja em ambiente rural ou urbano ${ }^{3}$.

Aspectos relacionados às atividades rurais apontam que as queixas de dor/desconforto musculoesquelético pontual e/ou multifocal dos trabalhadores possuem relação com a postura e movimento corporal adotado durante a jornada de trabalho. Igualmente, a postura inadequada do labor rural expõe o trabalhador aos riscos de desenvolver lesões ou doenças ocupacionais, principalmente na região lombar, cervical e membros superiores ${ }^{6-8}$.

Corroborando a nocividade dessa atividade, a Organização Internacional do Trabalho (OIT) afirma que o trabalho rural é significativamente mais perigoso que outras atividades ocupacionais e estima que milhões de agricultores sofram sérios problemas de saúde 9 . Dentre esses, encontram-se as dores musculares proveniente de traumas e lesões e/ou do sofrimento do aparelho osteomuscular e Lesões por Esforço Repetitivo (LER) ${ }^{10-13}$.

A jornada do trabalhador rural pode se estender por até 12 horas diárias e seis vezes na semana ${ }^{14}$, motivo que pode dificultar a participação dos trabalhadores em atividades externas ao seu ambiente laboral. Considerando que os adolescentes do meio rural estão expostos aos mesmos riscos em função das atividades ocupacionais, percebeu-se a necessidade de desenvolver ações 
de educação em saúde com essa população sobre os aspectos posturais para que ao se apropriarem de conhecimento possam exercer o autocuidado, bem como tornar-se um multiplicador de saber em suas famílias e comunidade.

No Brasil, as ações de educação em saúde tiveram suas raízes nas primeiras décadas do século $X X^{15}$. A educação sempre esteve fortemente presente no cotidiano do fazer em saúde e atualmente é uma prática fundamental no contexto do Sistema Único de Saúde (SUS). Segundo Pinafo, Nunes e Gonzáles ${ }^{16}$ a educação em saúde pode ser vista como um campo de práticas sociais estabelecidas pelos profissionais de saúde entre si, com a instituição e, sobretudo, com o usuário, no desenvolvimento cotidiano de suas atividades. É um processo educativo de construção de conhecimentos por meio de práticas que contribuem para aumentar a autonomia das pessoas no cuidado em saúde. Neste estudo, o embasamento das ações se deu mediado pela teoria transcultural de Leininger, que busca a manutenção/preservação; ajustamento/negociação e repadronização/reestruturação do conhecimento para o cuidado cultural.

De acordo com Leininger, a diferença no comportamento dos jovens possui relação com a sua base cultural, por isso é necessário saber sobre seu conhecimento para trabalhar o cuidado, pois as mudanças devem ser negociadas e a relação linear evitada ${ }^{17}$. 0 presente estudo teve por objetivo promover o autocuidado postural aos adolescentes do meio rural.

\section{Materiais e métodos}

$\infty \times \infty \times \infty \times \infty \times \infty \times \infty \times \infty \times \infty \times \infty \times \infty \times \infty \times \infty \times \infty \times \infty$

Trata-se de uma pesquisa-ação desenvolvida com estudantes do sétimo e oitavo ano de duas escolas, representadas pelas siglas E1 e E2, do meio rural de um município do Oeste de Santa Catarina (SC), no primeiro semestre de 2013. Pesquisa-ação trata-se de um tipo de pesquisa social com base empírica que intenciona desenvolver uma ação ou resolução de um problema coletivo no qual os envolvidos interagem de modo cooperativo e participativo. Dentro dessa concepção, o saber do especialista não é suficiente para ser aplicado às situações que precisam ser modificadas, por isso requer o estabeleciomento de um diálogo intercompreensivo com os agentes do saber popular ${ }^{18}$.

Para Tripp ${ }^{19}$ a pesquisa-ação é uma forma de investigação-ação que utiliza técnicas de pesquisas para construir uma ação coletiva, que segue um ciclo de ir e vir, ou seja, de agir (ir) na prática e investigar (vir) se a ação foi adequada para qualificar a práxis.

Para conhecer as posturas adotadas pelos jovens nas atividades investigadas, 0 instrumento de coleta de dados utilizado foi um questionário, aplicado com os 65 alunos das escolas E1 e E2, que, individualmente, responderam as questões: Qual a postura que vocês adotam quando permanecem sentados na cadeira da sala de aula e da sua casa? Qual a postura que vocês adotam quando se mantém sentados escrevendo no caderno? De que maneira vocês transportam a sua mochila/pasta/ bolsa para escola? De que forma vocês pegam e levantam um objeto pesado no chão? De que forma vocês pegam e levantam um objeto leve do chão?

Após a demonstração dos movimentos de cada postura questionada, as mediadoras das oficinas (docente e bolsista de iniciação científica), demonstravam as posturas adequadas para cada situação investigada. Tendo sempre como base norteadora do processo de apreensão do conhecimento a teoria do cuidado cultural de Leininger, em que os conhecimentos existentes dos adolescentes eram mantidos/preservados quando corretos; ajustados/negociados quando parcialmente corretos e repadronizados/reestruturados quando impróprios. Ressaltando que o conhecimento no momento das oficinas mantidos ou (re)construídos deveriam estar congruentes com a cultura ${ }^{20}$.

A análise univariada dos dados coletados foi realizada por meio de estatística descritiva com verificação de frequências relativa e absoluta. Os dados foram expostos em uma tabela com os respectivos resultados e seu percentil a fim de evidenciar os achados e auxiliar na comunicação ${ }^{21}$.

Para atender os compromissos éticos, o projeto de pesquisa foi autorizado pela Secretaria de Estado do Desenvolvimento Regional - Gerência de Educação para o desenvolvimento do estudo com os adolescentes e aprovado pelo Comitê de Ética e Pesquisa da Universidade Comunitária da Região de Chapecó (Unochapecó), sob o parecer 
$274 / 12$. Para os estudantes, foi elaborado um termo de assentimento e, por serem menores de idade, foi solicitado o consentimento de seus pais ou responsáveis por meio da assinatura do Termo de Consentimento Livre e Esclarecido (TCLE).

\section{Resultados e Discussão}

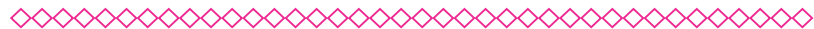

Na E1 participaram do estudo 33 alunos e na E2, 32 alunos. Os adolescentes participantes tinham entre 11 e 16 anos. Na E1,63,88\% eram do sexo masculino e $36,11 \%$ do sexo feminino e na E2, 46,34\% e 53,65\%, respectivamente. Sobre a apresentação das posturas demonstradas pelos adolescentes do meio rural (Tabela $\mathbf{1}$ ), os resultados acusam movimentos posturais que requerem a manutenção/preservação; o ajustamento/negociação e repadronização/reestruturação do conhecimento para o cuidado cultural.

A respeito das formas do cuidado cultural Leininger e McFarland ${ }^{20}$ esclarecem que se aplica da seguinte forma: preservação/manutenção do cuidado cultural, quando ações e decisões profissionais auxiliam indivíduos de uma determinada cultura a preservar e/ou manter valores culturais importantes acerca de cuidado à saúde e bem-estar; acomodação/negociação do cuidado cultural, quando as ações profissionais ajudam as pessoas de uma determinada cultura a adaptarem-se ou a negociarem com as outras um resultado de saúde favorável; repadronização/restruturação do cuidado cultural caracterizam-se pelas ações e decisões profissionais que ajudam os indivíduos a reorganizar e modificar seu modo de vida mantendo o respeito à suas crenças e valores culturais, porém com um novo padrão de cuidado mais benéfico à sua saúde.

Em relação ao modo de permanecer sentado na cadeira da sala de aula e em casa, 84,6\% dos alunos adotam uma postura inadequada, bem como na manutenção da posição sentada "escrevendo no caderno", em que 81,5\% o fazem de forma inapropriada. De acordo com Ritter ${ }^{22}$ e Steffenhagen, a postura correta para a posição sentada na cadeira foi considerada aquela em que o escolar sentava sob os ísquios, com a coluna apoiada no encosto da cadeira, quadris e joelhos com angulação em torno de $90^{\circ}$ à $100^{\circ}$ e pés apoiados no solo. 0 mesmo critério foi adotado para a posição sentada escrevendo, sendo considerado incorreto sentar com o tronco fletido, ou sob o sacro.

Quanto à postura na sala de aula, ao avaliar as alterações posturais de estudantes do sexo feminino, o estudo apontou que apenas $10 \%$ das meninas sentavam-se corretamente com os pés e costas apoiadas, enquanto que quase $90 \%$ das estudantes utilizavam outras posturas, classificadas como incorretas $^{23}$. A respeito da postura sentada ao escrever, os alunos do ensino fundamental pouco utilizam os critérios biomecânicos de adequação da postura para essa atividade 22,24 .

Zapater ${ }^{4}$ relatam que a adaptação do ambiente físico utilizando mobiliário ajustável às particularidades de cada indivíduo é uma forma de minimizar os problemas da postura sentada, contudo, isoladamente não é suficiente para resolver essa questão, sendo necessária a adoção de programas de treinamentos preventivos para minimizar os riscos e maus hábitos posturais.

Outro estudo demonstrou que $55 \%$ dos adolescentes de uma escola estadual de Rondônia apresentavam algum tipo de desvio postural e a maneira de sentar na sala de aula foi relacionada como uma variável de significância relacionada à escoliose ${ }^{25}$. A postura inadequada e viciosa adotada pelos adolescentes pode ser imposta pelo mobiliário escolar. Os resultados desta investigação demonstram que há um desajuste do mobiliário da escola à população estudantil, evidenciado pelas atitudes corporais que mantêm durante a sua utilização ${ }^{26}$.

Evidenciou-se que em ambas as escolas são utilizadas mochila, pasta e bolsa para carregar o material escolar. Contudo, observou-se uma postura inadequada na demonstração do uso desses objetos, principalmente no uso da mochila de costas, o que pode acarretar, segundo Candotti, Noll e Roth, ${ }^{27}$ sobrecarga na região dorsal e ocasionar hipercifose torácica, como compensação, hiperlordose lombar e cervical.

A forma considerada ideal para transportar o material escolar é aquela que utiliza a bilateralidade no uso da mochila, pasta e bolsa, com uso de alças longas, que não ultrapassem a crista ilíaca ${ }^{22}$. 
Em estudo que estimou a prevalência de alterações posturais laterais e anteroposteriores em adolescentes do sexo feminino, foi observado que $55,7 \%$ das meninas utilizavam a postura correta com a mochila ou bolsa, isto é, vestindo as duas alças ou com ela transpassada, e 44,4\% usavam da forma incorreta, ou seja, com a alça em um ombro só $^{23}$.

Objetivando verificar a existência de associação entre escoliose e o modo de transporte do material escolar no ensino fundamental Freire ${ }^{28}$ identificou que as anormalidades mais frequentes (hipercifose, hiperlordose e escoliose) nos jovens, provavelmente, estão associadas aos hábitos posturais inadequados no local de realização de tarefas, na forma e local utilizado para carregar o material escolar, entre outros.

Acerca da forma como os alunos pegam e levantam objetos pesados do chão, a maioria dos adolescentes o faz da forma menos recomendada, isto é, agacham-se sem flexionar os joelhos e a coluna ereta, sendo que apenas $23 \%$ executam do modo correto. A respeito do modo de pegar e levantar um objeto leve do chão, somente $12,3 \%$ demonstraram adotar a forma mais adequada para essa atividade. A atividade de levantar objetos do solo é considerada como fator de risco para degeneração do disco intervertebral e para desordens musculoesqueléticas lombares ${ }^{28}$.

Considerando que o meio escolar tem influência nas alterações posturais das crianças e que a recorrência da postura inadequada nesse ambiente pode tornar-se permanente na vida adulta ${ }^{30}$, parece pertinente identificar as alterações posturais em escolares e suas alterações compensatórias precocemente pelo fato da instalação, progressão e agravamento postural durante a adolescência e a vida adulta e o comprometimento da postura nessas fases ${ }^{31}$. Nessa ótica, a importância da educação postural como prática preventiva no ambiente escolar, prevenindo alterações posturais e deformidades, é fundamental para diminuir a ocorrência de vícios posturais e evitar as alterações ${ }^{32}$.

Rebolho, Casarotto e Amado-João ${ }^{33}$ verificaram os efeitos de um programa de educação postural comparando duas estratégias de ensino, sendo uma com utilização de história em quadrinhos e outra com experiência prática de posturas cor- retas e incorretas. Participaram do estudo 80 estudantes de segunda e terceira séries, com idade entre 7 e 11 anos, divididos em dois grupos iguais, sendo que foram trabalhadas as posturas corretas em pé, sentado, transportando mochilas, abaixando, mudando objetos de lugar e forma de dormir. Houve aumento significativo no aprendizado e memorização dos hábitos posturais corretos em todas as variáveis estudadas em ambos os grupos, não havendo diferença significativa entre as duas estratégias educativas.

Outro estudo que avaliou a eficácia de um programa de educação em saúde sobre o conhecimento relacionado à postura sentada com 71 estudantes da primeira série do ensino fundamental em escolas estadual, municipal e particular, em Bauru (SP), constatou que o programa se mostrou eficaz, aumentando os conhecimentos dos escolares a respeito da postura em todas as escolas estudadas. Os autores ainda reforçam que a escola é um espaço vantajoso para realizar ações preventivas e de educação em saúde postural que contribuam para o planejamento de medidas que visem à manutenção, melhora e promoção do bem-estar físico dos escolares ${ }^{4}$.

No presente estudo, a mediadora demonstrou aos adolescentes os movimentos corporais mais adequados para desenvolver as atividades propostas, com a intenção de ajustar e repadronizar o conhecimento sobre as formas de sentar na cadeira; de carregar a mochila/pasta/bolsa e de pegar um objeto pesado/leve no chão, objetivando minimizar os agravos na coluna vertebral; o encurtamento muscular; as alterações na curvatura da coluna e o comprometimento do retorno venoso dos membros inferiores. Além disso, foi negociado que o peso dos objetos carregados dentro da mochila não deve ultrapassar $10 \%$ do peso corporal dos alunos; a postura mais adequada para andar de bicicleta; a melhor posição corporal para dormir e os cuidados prévios à prática de atividade física.

Após a exposição pela mediadora das posturas corporais adequadas, solicitou-se aos adolescentes que repetissem os movimentos apresentados. Nesse momento houve, em alguns casos, a negociação/repadronização de atitudes posturais que ainda não haviam sido compreendidas por alguns adolescentes. Além disso, foi solicitado (firmado 
um pacto de compromisso) que os estudantes demonstrassem para seus familiares o que tinham vivenciado nas oficinas.

A estratégia de trabalhar com os jovens na perspectiva de multiplicadores do conhecimento acerca do tema educação postural possibilita a qualificação dos adolescentes para o enfrentamento de vulnerabilidades em saúde ${ }^{34}$. Além disso, é um meio de socializar as informações, estimulando uma conexão de redes sociais que possam ser corresponsáveis ${ }^{35}$.

A formação de agentes mirins de saúde parece um método eficiente para multiplicar o conhecimento, na medida em que as crianças praticam o que aprendem ${ }^{36}$. Fonseca ${ }^{37}$ avalia de forma positiva a qualificação de crianças e jovens para atuarem como multiplicadores de informações preventivas e de promoção à saúde, pois eles passam a exigir o cuidado de suas famílias e da comunidade sobre o tema abordado, constatando-se mudanças de hábito de outras pessoas orientadas por esses multiplicadores.

Um estudo de Foltran ${ }^{38}$ avaliou os efeitos de um programa educacional de cuidados com a coluna sobre o nível de conhecimento de escolares quanto à prevenção de dor na coluna vertebral e controu ccom a participação de 392 estudantes da quarta à oitava série de uma escola estadual, sendo que 114 foram avaliados após dois anos do término do programa. Os resultados demonstraram que o programa preventivo aumentou o nível de conhecimento dos estudantes e se manteve após dois anos de sua implementação, destacando que a aquisição de conhecimento é importante para a adoção de hábitos saudáveis para prevenção de problemas na coluna vertebral.

Um estudo de revisão sistemática e meta-análise para verificar as evidências sobre intervenções preventivas para cuidados posturais em crianças e adolescentes demonstrou que programas de intervenções aumentaram significativamente 0 comportamento preventivo e o conhecimento de estudantes quanto à postura ${ }^{39}$.

Ao se trabalhar com programas educativos é necessária a ação conjunta e integrada de diferentes atores e esferas, incluindo profissionais de diferentes áreas, o governo e a comunidade. Quando se quer modificar um padrão vicioso e, neste caso, se tratando de hábitos posturais, a sensibilização e mobilização de pequenos grupos, com ações conjuntas e participativas, podem produzir mudanças que, gradativamente se multiplicarão e refletirão no todo. Neste caso, a escola é um local importante para iniciar este trabalho com metas a curto, médio e longo prazo, pois permite o acompanhamento das intervenções por um período maior de tempo, já que os adolescentes a frequentam diariamente ${ }^{2}$

O exercício da prática de educação em saúde pressupõe abertura, disponibilidade para ouvir o outro, horizontalidade na relação interpessoal e na ação educativa em si, pois o ato participativo é humanizante. Nesse sentido, parece-nos que o campo da saúde carece de um discurso e de uma prática transformadora, mediados pela participação do sujeito em todo processo educativo. Afinal, a premissa básica daqueles que realizam esse processo é propiciar o fortalecimento pessoal dos seres humanos com quem interagem para que estes façam escolhas saudáveis e que nesse processo exerçam o autocuidado. 0 essencial é apoiar o ser humano a ajudar-se, é fazê-lo agente de sua transformação ${ }^{40}$.

0 fato de que ao participar de programas preventivos os estudantes agregaram conhecimentos que servem de subsídio para a mudança de hábitos posturais inadequados, justifica a utilização das abordagens com foco na educação em saúde, visto que são de baixo custo para o sistema e constituem-se, ainda, como estratégias eficazes de transformação, em que os sujeitos que participam das atividades podem constituir-se em multiplicadores desses novos conhecimentos, educando seus pares e contribuindo para o bem-estar das pessoas nos ambientes em que frequentam.

\section{Considerações finais}

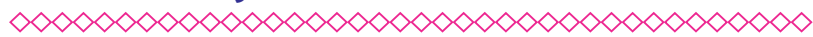

A pesquisa demonstrou que a maioria dos estudantes apresentou hábitos posturais inadequados, destacando a postura sentada na cadeira, a forma de carregar material escolar e de levantar objetos leves e pesados do chão, fato que evidencia a importância do desenvolvimento de atividades educativas que abordem tais questões. 
Medidas preventivas pontuais foram realizadas, mas são necessárias novas intervenções com esse público para evitar que desenvolvam problemas posturais futuros.

Além disso, no processo educativo, devese considerar os aspectos culturais dos sujeitos envolvidos. Para tanto, torna-se fundamental o conhecimento do ambiente onde vivem, estudam e trabalham. Igualmente, a busca por soluções e melhorias da postura corporal precisa emergir dos participantes, com o apoio de profissional mediador.

\section{Referências}

$\infty \times \infty \times \infty \times \infty \times \infty \times \infty \times \infty \times \infty \times \infty \times \infty \times \infty \times \infty \infty$

1. Magge DJ. Avaliação musculoesquelética. 4. Ed. São Paulo: Manole, 2005.

2. Braccialli LMP, Vilarta R. Aspectos a serem considerados na elaboração de programas de prevenção e orientação de problemas posturais. Revista Paulista de Educação Física 2000; 14:159-71.

3. Kisner C, Colby, Lynn A. Exercícios terapêuticos: fundamentos e técnicas. 5. ed. São Paulo: Manole, 2009.

4. Zapater AR, Silveira DM, Vitta A, Padovani CR, Silva JCP. Postura sentada: a eficácia de um programa de educação para escolares. Ciênc. saúde coletiva. 2004; 9(1): 191-199.

5. Steffenhagem MK. Manual da Coluna: mais de 100 exercícios para você viver sem dor. Curitiba: Estética Gráphica, 2003.

6. Freitas CS. Análise ergonômica da atividade com pulverizador costal manual na cultura do café no município de Caratinga, MG. Dissertação (mestrado) - Centro Universitário de Caratinga, 2006.

7. Oliveira CC. A ergonomia na atividade leiteira: avaliação das condições de trabalho dos ordenadores na região de Campo Mourão - P.R. Dissertação (mestrado) -Universidade Tecnológica Federal do Paraná, 2011.

8. Costa CKL, Lucena NMG, Tomaz AF, Másculo FS. Avaliação ergonômica do trabalhador rural: enfoque nos riscos laborais associados à carga física. Gestão Prod

9. Oper Sis. $2011 ; 6(2): 101-12$.

10. Teixeira MLP, Freitas RMV. Acidentes do trabalho rural no interior paulista. São Paulo Perspect. 2003;17(2):81-90.

11. Levigard YE, Rozemberg B. A interpretação dos profissionais de saúde acerca das queixas de "nervos" no meio rural: uma aproximação ao problema das intoxicações por agrotóxicos. Cad. Saúde Pública. 2004; 20( 6 ): 1515-1524.

12. Organização Internacional Do Trabalho - OIT. Módulos de autoaprendizagem sobre Saúde e Segurança no Trabalho Infantil e Juvenil. Brasília: Ministério da Saúde, 2006.

13. Falzon, P. Ergonomia. São Paulo: Blucher, 2007.

14. Lucca SR, Cortez mas, Tosseto T. A percepção dos trabalhadores sobre os riscos de distúrbios osteomusculares ao trabalho na produção de Rosas. Revista Espaço de Diálogo e Desconexão. 2011; 4 (1).

15. Veiga MM, Duarte FJCM, Meirelles LA, Garrigou A, Baldi I. A contaminação por agrotóxicos e os Equipamentos de Proteção Individual (EPIs). Revista Brasileira de Saúde Ocupacional. 2007; 32 (116): 57-68.

16. Gomes LB, Merhy EE. Compreendendo a educação popular em saúde: um estudo na literatura brasileira. Cad. Saúde Pública. 2011; 27(1): 7-18.

17. Pinafo Elisangela, Nunes Elisabete de Fátima Polo de Almeida, González Alberto Durán. A educação em saúde na relação usuário-trabalhador no cotidiano de equipes de saúde da família. Ciênc. saúde coletiva. 2012; 17(7): 1825-1832.

18. Leopardi MT. Teoria e método em assistência de enfermagem. 2. ed. Florianópolis: Soldasoft, 2006.

19. Thiollent M. Metodologia da pesquisa-ação. 15. ed. São Paulo: Cortez, 2007.

20. Tripp D. Pesquisa-ação: uma introdução metodológica. Educação e Pesquisa. 2005; 31(3):443-6 
21. Leininger MM, Mcfarland MR. Culture care diversity and universality: a worldwide nursing theory. 2. ed. Boston: Jones and Bartlett, 2006.

\section{Marconi MA, Lakatos EM. Técnicas de}

Pesquisa: planejamento e execução de pesquisas, amostragens e técnicas, elaboração, análise e interpretação de dados. 5 ed. São Paulo: Atlas, 2002.

\section{Ritter ALS. Postura corporal ao sentar e} transportar material escolar. Tese (doutorado) Escola de Educação Física da Universidade Federal do Rio Grande do Sul, 2009.

\section{Detsch C, Luz AMH, Candotti CT, Oliveira DS} de, Lazaron F, Guimarães LK et al. Prevalência de alterações posturais em escolares do ensino médio em uma cidade no Sul do Brasil. Rev Panam Salud Publica. 2007; 21(4): 231-238.

25. Moreira S. Características da postura corporal de escolares da rede municipal de ensino de Porto Alegre. Dissertação (mestrado) - Escola de Educação Física da Universidade Federal do Rio Grande do Sul, 2008.

\section{Politano RC. Levantamento dos desvios} posturais em adolescentes de 11 a 15 anos em escola estadual do município de Cacoal-RO. Dissertação (Mestrado) - Universidade de Brasília, 2006.

27. Barbosa AF. Avaliação da Influência do Mobiliário Escolar na Postura Corporal em Alunos Adolescentes. Dissertação (Mestrado) Universidade do Minho. Dissertação de Mestrado em Engenharia de Produção e Sistemas Área de Especialização Engenharia Humana, 2009.

28. Candotti C, Roth E, Noll M. Avaliação do peso e do modo de transporte do material escolar em alunos do ensino fundamental. Revista Paulista de Pediatria. 2012; 30 (1): 100-6.

29. Freire TCPB. Prevalência de Escoliose em Alunos do Quinto ao Nono Ano, na Faixa Etária de 10 a 15 Anos, Matriculados nas Escolas Públicas Municipais de Guaramiranga/CE no Primeiro Trimestre de 2008. Dissertação (mestrado) Escola Nacional de Saúde Pública Sérgio Arouca, 2008.
30. Torre ML, Aguiar TMC, Araújo LD, Loss JF. Cálculo das forças internas na coluna lombar durante levantamento de carga através da dinâmica inversa. 2005.

\section{Contri DE, Petrucelli A, Perea DCBNM.} Incidência de desvios posturais em escolares do $2^{\circ}$ ao $5^{\circ}$ ano do ensino fundamental. Conscientiae Saúde. 2009;8(2):219-24.

32. Santos CIS, Cunha ABN. Braga VP, Saad IAB. Ribeiro MÂGO, Conti PBM et al. Ocorrência de desvios posturais em escolares do ensino público fundamental de Jaguariúna, São Paulo. Rev. paul. Pediatr. 2009; 27(1):74-80.

33. Rego A, Scartoni FR. Alterações posturais de alunos de $5^{\mathrm{a}}$ e $6^{\mathrm{a}}$ séries do Ensino Fundamental. Fit Perf J. 2008; 7(1): 10-15.

34. Rebolho MCT, Casarotto RA, Amado JSMA. Estratégias para ensino de hábitos posturais em crianças: história em quadrinhos versus experiência prática. Fisioter. Pesqui. 2009; 16(1): 46-51.

35. Calderon CESC, Bittelbrunn ADWB. Vulnerabilidade na adolescência: promoção à saúde a partir do autocuidado. Revista Caminhos. 2011; 2 (3): 91-100.

36. Alves MS. et al. Adolescente como agente Multiplicador em Educação Sexual. Revista Integrativa e saúde e educação - REVISE. 2010; 2(2): 2179-6572.

37. Garcia RN, Almeida EB, Distéfano EG, Farenzena E, Menegol R, Menegat D, et al. Agentes mirins de saúde: uma estratégia para formar multiplicadores de conhecimento. Rev SulBrasileira Odontologia. 2009;6(1):13-9.

38. Fonseca FF. A Escola como espaço facilitador para promoção da saúde e prevenção de riscos. UNIPLI, 15 a 17 de maio de 2008, Niterói/RJ. Encontro Nacional de Ensino de Ciências da Saúde e do Ambiente.Anais do Encontro Nacional de Ensino de Ciências da Saúde e do Ambiente 15 a 17 de maio de 2008 Niterói/RJ.

39. Foltran Fabiana A., Moreira Roberta F. C., Komatsu Mariana O., Falconi Mariana F., Sato Tatiana O. Effects of an educational back care 
program on Brazilian schoolchildren's knowledge regarding back pain prevention. Rev. bras.

fisioter. 2012; 16( 2 ): 128-133.

40. Calvo-Muñoz I, Gómez-Conesa A, SánchezMeca, J. Preventive physiotherapy interventions for back care in children and adolescents: a metaanalysis. BMC Musculoskeletal Disorders. 2012; 13 (1): 152, 2012

41. Alvim Neide Aparecida Titonelli, Ferreira Márcia de Assunção. Perspectiva problematizadora da educação popular em saúde e a enfermagem. Texto contexto - enferm. 2007 Jun; 16(2): 315-319 


\section{Anexos}

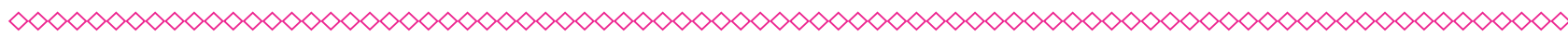

Tabela 1 - Apresentação das posturas demonstradas pelos adolescentes do meio rural na oficina da pesquisa-ação. Chapecó-SC, 2013

\begin{tabular}{|l|c|c|}
\hline Postura sentada na cadeira da sala de aula e de casa & $\mathbf{N}$ & $\%$ \\
\hline Executam a atividade de forma adequada & 10 & 15,4 \\
Executam a atividade de forma inadequada & 55 & 84,6 \\
\hline Postura sentada escrevendo no caderno & $\mathbf{N}$ & $\%$ \\
\hline Executam a atividade de forma adequada & 12 & 18,5 \\
Executam a atividade de forma inadequada & 53 & 81,5 \\
\hline Postura ao transportar o material escolar & $\mathbf{N}$ & $\%$ \\
\hline Carregam o material na mão & 3 & 4,6 \\
Executam a atividade de forma adequada quando usam pasta de alça longa, alça & 8 & 12,3 \\
curta ou sem alça & & \\
Executam a atividade de forma inadequada quando usam pasta de alça longa, alça & 3 & 4,6 \\
curta ou sem alça & 51 & 78,5 \\
\hline Executam a atividade de forma inadequada quando usam mochila & $\mathbf{N}$ & $\%$ \\
\hline Postura adotada ao pegar e levantar um objeto pesado no chão & 15 & 23 \\
\hline Executam a atividade de forma adequada & 50 & 77 \\
\hline Executam a atividade de forma inadequada & $\mathbf{N}$ & $\%$ \\
\hline Postura adotada ao pegar e levantar um objeto leve no chão & 8 & 12,3 \\
\hline Executam a atividade de forma adequada & 57 & 87,7 \\
\hline Executam a atividade de forma inadequada & 65 & $100 \%$ \\
\hline Total & & \\
\hline
\end{tabular}

